\title{
Design of a Medical Image Database with Content-Based Retrieval Capabilities
}

\author{
Juan C. Caicedo, Fabio A. González, Edwin Triana, and Eduardo Romero \\ Bioingenium Research Group \\ Universidad Nacional de Colombia \\ \{jccaicedoru, fagonzalezo, emtrianag, edromero\}@unal.edu.co \\ http://www.bioingenium.unal.edu.co
}

\begin{abstract}
This paper presents the architecture of an image administration system that supports the medical practice in tasks such as teaching, diagnosis and telemedicine. The proposed system has a multi-tier, web-based architecture and supports content-based retrieval. The paper discusses the design aspects of the system as well as the proposed contentbased retrieval approach. The system was tested with real pathology images to evaluate its performance, reaching a precision rate of $67 \%$. The detailed results are presented and discussed.
\end{abstract}

Keywords: content-based image retrieval, medical imaging, image databases.

\section{Introduction}

Nowadays medical environments generate a large number of digital images to support clinical decisions, the Geneve University Hospital reported a production rate of 12.000 daily images during 2,002 [1]. The problem of archiving those medical image collections have been addressed with different solutions such as PACS1 [23] or modular and specialized systems for image databases [45]. Effectiveness of those systems may be critical in clinical practice [6] since they are responsible for storing medical images in a dependable way. Besides, these systems must allow users to efficiently access this information.

Traditional medical image database systems store images as a complementary data of textual information, providing the most basic and common operations on images: transfer and visualization. Usually, these systems are restricted to query a database only through keywords, but this kind of queries limit information access, since it does not exploit the intrinsec nature of medical images. On the other hand, a medical image database system must have a flexible architecture along with a wide variety of functionalities supporting clinical, academic and research tasks [7. Medical users must count on a set of automated and efficient tools, which permits efficient access to relevant information.

A recent approach to medical image database management is the retrieval of information by content, named Content-Based Image Retrieval (CBIR) 1]. This

${ }^{1}$ Picture Archiving and Comunication Systems.

D. Mery and L. Rueda (Eds.): PSIVT 2007, LNCS 4872, pp. 919-931, 2007.

(C) Springer-Verlag Berlin Heidelberg 2007 
kind of systems allow evaluation of new clinical cases so that when similar cases are required, the system is able to retrieve comparable information for supporting diagnoses in the decision making process. Several systems have been developed following this approach such as ASSERT [8], IRMA 9], FIRE [10] among others 1112 13. The main idea of this kind of systems is to achieve adequate support to physicians in reaching their medical goals [7].

This paper presents the design, implementation and evaluation of an image database system for the Telemedicine Centre at the National University of Colombia. Physicians asociated to the Telemedicine Centre have attended to cases in dermathology, pathology and radiology, related to general and tropical diseases in remote places since 2,002. The number of received cases has been steadily increasing and each case has many images associated with different types of metadata. Presently, this amounts to an average of 100,000 digital images per year to be managed.

The architecture of the proposed image database system is multi-tier and allows easy mantenance and extensibility. This system is a web-oriented service platform to explore any image collection from anywhere, following state-of-theart security requirements. The system is capable of processing and storing any type of medical image format, including DICOM 2 , TIFF or any conventional digital photography. In addition, the proposed system includes a module for content-based retrieval, configurable with different features and metrics. The CBIR module performance was assessed with an annotated histopathology image collection composed of 1,502 basal-cell carcinoma images. The reminder of this paper is organized as follows. In section 2, we define the requirements for the image database system. In section 3 , a state of the proposed methods for contentbased image retrieval are presented. The system architecture is then analyzed in section 4 and section 5 presents results of the experimental evaluation. Finally, some concluding remarks are presented in section 6 .

\section{Defining Requirements}

Many technologies are currently available to capture medical images and support clinical decisions, but there exists little work to develop clever management systems for that amount of visual information. The required system must support the medical workflow, which includes clinical, academic and research tasks. Figure1illustrates a general overview of the required system which must provide support to image capturing, storing and methods for allowing similarity queries.

\subsection{Functional Requirements}

There exist different devices and methods for image capture in a health center. A medical case may require either only one picture or many slides. Whatever the number of images to store is, the system must provide a standard interface that permits uploading of any number of images. Each image can also have different

\footnotetext{
${ }^{2}$ Digital Imaging and Communication in Medicine.
} 


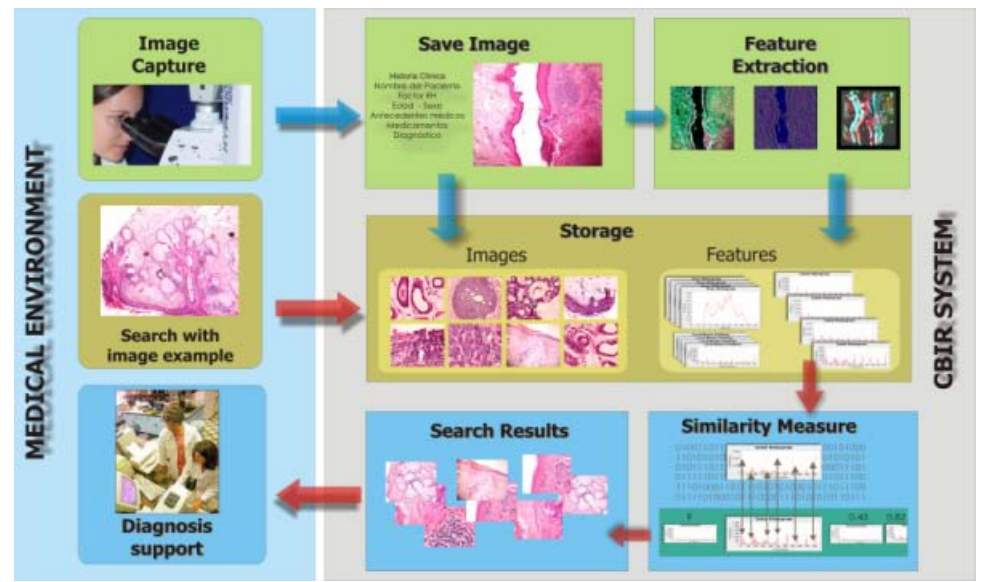

Fig. 1. Overview of the image database system architecture

annotations or textual descriptions that need to be stored together with the visual raw data. The system must provide the possibility of associating textual metadata and queries using available key-words, e.g DICOM attributes.

Once images are stored, physicians might explore a particular image collection for retrieving specific cases or simply for taking an overview on the whole collection. This exploration could be structured by metadata, filtering image results with some textual conditions, for instance dates or image modality. As a high number of results may be delivered in the exploration process, images should be presented in pages with a specific number of items per page. Image details must be shown at the user's request. The system must allow the downloading of images with their associated metadata.

Since medical images are characterized by their particular visual patterns, the system must support queries to the image collection using a given pattern image, which then triggers the process of delivering a similar image set. Querying by similarity is thus a very desirable and useful property of the system for physicians, to find images with similar patterns. CBIR systems have many benefits in a medical environment [1] e.g. a computer-aided diagnosis tool. The system must provide tools for automatic image processing, with specialized image analysis if needed, to support queries by similarity.

\subsection{Technical and Architectural Requirements}

Design of such an image database system must consider extensibility and scalability. Extensibility through a modular architecture that incorporates new functionalities and capabilities, scalability by allowing new resources to be naturally pluged to the system in order to extend the service, covering more users or more data.

The system must be capable of managing any type of digital image format, after requirements for any medical speciality have been established; for instance, 
in dermathology, where resolution is less crucial, the common acquisition method is a digital picture, commonly stored in a JPEG format. In pathology, a digital camera coupled to the microscopy allows users to capture images in TIFF, JPEG or BMP format, while the standard format for radiology is DICOM [5].

Importantly, the image database system should not be designed for personal use as a desktop software. Main core system functionalities must be executed in a high-performance computing platform, allowing concurrent users through network services. In addition, images and medical data can not be exposed to unauthorized access so that the system must provide an authentication security module.

The system has to be devised for allowing access through the web [14, but keeping in mind that the main uses of a medical image database is to support diagnosis and the associated academic and research activities. In addition, search of relevant images should be an easy process, that is to say, the system must provide content-based retrieval mechanisms such as query by example and relevance feedback. [1].

\section{Content-Based Retrieval Methods}

The content-based image retrieval module allows users to obtain a set of similar images from an example image, i.e making a query-by-example request [15. The example image is analyzed and compared with the other database images and the most similar images are identified in a ranking process. In this process, image features and similarity measures are used.

\subsection{Image Retrieval Model}

The fisrt goal here is to define how images have to be compared. A Medical image is a two, three or four dimensional discrete signal with information about colors, luminance, volume or time in one scene. Therefore, images are difficult to compare because each one is an object, provided with complex properties and different structure. Other technical aspects make images difficult to compare, such as different widths or heights, color representations and formats. A very convenient manner to face these difficulties consists of using a statistiscal frame: images are modeled as random variables because they are the product of a stochastic process. Then, many stastistical measurements can be obtained from images and used as characteristic features. On the other hand, image analysis is required for structuring visual data information. Common features computed for such a tasks comprise a broad range of possibilities [16], but the very basic ones are color distribution, edges and textures.

Formally, the feature extraction process is defined as:

Definition 1. Let $I$ be the image collection into the database. Let $F_{k}$ be the space defined for a feature $k$. The extraction process for a feature $k$ is defined as a function between $I$ and $F_{k}$ :

$$
E_{k}: I \longrightarrow F_{k}
$$


There exists a feature space onto which images are mapped when a specific feature is extracted so that all images are now represented by their corresponding features in that space. In addition, many feature spaces have to be supported by the image database system and different measurement strategies must be defined for each. If we assume that those feature spaces are metric spaces, distance functions can be devised for determining the similarity degree of images in each of such metric spaces. A metric space is a tuple $\left(F_{k}, M_{k}\right)$, where $F_{k}$ is a set and $M_{k}$ a metric on $F_{k}$ as follows:

Definition 2. Let $F_{k} \times F_{k}$ be the cartesian product between features of the same space. Let $M$ be a metric that calculates the similarity between a pair of given features, then:

$$
M_{k}: F_{k} \times F_{k} \longrightarrow \mathbb{R}
$$

such that:

1. $M_{k}(x, y) \geq 0$. Non-negativity

2. $M_{k}(x, y)=0$, if and only if $x=y$. Identity

3. $M_{k}(x, y)=M_{k}(y, x)$. Simetry

4. $M_{k}(x, z) \leq M(x, y)+M_{k}(y, z)$. Triangle inequality

Definition 2 permits to introduce an order relationship between images using a feature $k$ and a metric $M_{k}$. Previous definitions allow to perform image comparisons using one feature an one metric. However, better comparisons may be achieved using many features and a linear combination of different metrics, as follows:

Definition 3. Let $x, y \in I$ be images. Let $E_{k}$ be the feature extraction function of a feature $k$ and $M_{k}$ be a metric in the feature space $F_{k}$. A similarity function for different features is defined as the linear combination of metrics $M_{k}$ with importance factors $w_{k}$ :

$$
s(x, y)=\sum_{k} w_{k} M_{k}\left(E_{k}(x), E_{k}(y)\right)
$$

\subsection{Feature Extraction}

Yet it is desirable to match image features and semantic concepts, most image processing techniques actually computes information at the very basic level. This is the well known semantic gap, and there are different approaches to bridge it, many of them including prior information about the application domain [17]. In this work we use features corresponding to a perceptual level, without any conceptual structure, named low level features. These features were selected both to evaluate extensibility of the proposed architecture and to assess performance regarding medical image retrieval. We select histogram features such as:

- gray scales and color histogram

- local binary partition

- Sobel histogram 
- Tamura textures

- invariant feature histogram

that have been previously used for content-based retrieval in different scenarios, and their details are described in 1819.

\subsection{Similarity Measures}

Features previously described define a set of feature spaces, and each image into the database is mapped onto each of those spaces. We assume that each feature space is characterized by a special topology, requiring a specific metric. We used the following metrics as candidates to make measurements in each feature space:

- Euclidean distance

- histogram intersection

- Jensen-Shannon divergence

- relative bin deviation

- Chi-square distance

In general, those metrics have been defined to calculate the similarity between two probability distributions [18, since features defined in this work are global histograms. The most appropriate metric for each feature space is found by experimental evaluation as described in section 5 .

\subsection{Retrieval Algorithm}

In order to find the set of images most similar to a query image, it is required a retrieval algorithm that ranks images. This algorithm uses metric information to sort images out in decreasing order from the most similar. The retrieval algorithm receives as parameters the image query and the set of metrics, features and importance factors to be applied. Then, the algorithm evaluates the similarity between each database image and the query image. This evaluation calculates each metric with its corresponding feature and importance score and integrates over all results to provide a unique similarity score per item. When all images are evaluated, results are sorted by similarity score. Although all images are ranked, only the $n$-top images of the ranking are presented. The $n$ parameter can be configured, and the user could request additional results, if needed.

This algorithm can compute a single metric or a linear combination of them, since it receives as parameter the set of metrics to be applied, the feature extraction functions and the importance factors associated to each metric.

\section{Proposed Architecture}

The proposed image database architecture is based on the Java 2 Enterprise Edition (J2EE) 20, that provides a multitiered and a distributed application model. In this model, each tier (also called layer) has a particular function in 
order to guarantee easy maintenance, extensibility and low coupling. Each tier offers services to other tiers, e.g. the Persistent tier provide support to retrieve and store results of the Bussines tier, as well as the Bussines tier processes information fed to the $W e b$ tier. J2EE also supports transactions, web-services and provides a well defined security scheme to control the access from local and remote clients through a rol-based security model for Enterprise Java Beans (EJB) and web components.

\subsection{Architecture}

System architecture is composed of five main layers, a strategy which allows dividing processing responsabilities, data management and verification. The global model of the architecture can be viewed in Figure 2. As said before, each tier has a particular function and the loose interaction between the tiers results in a system with the whole functionality. Each layer is hereafter described:

1. Client Tier: It contains the Graphical User Interface (GUI) which allows interaction with the system and visualization of images and data. This tier has two client types: the web client who uses the internet browser and applets to access the system through the $W e b$ tier; and the standalone client that can be a remote application using $\mathrm{RMI}^{3}$ through JND 4 to access the J2EE server.

2. Web Tier. It has Java Server Pages (JSP) and servlets. This tier processes requests and builds responses based on the Bussines tier results. This layer uses a local interface to invocate logical methods.

3. Bussines Tier. This is the system core, actually composed by Enterprise Java Beans (EJB) and plain Java classes. There are two types of EJBs: the Session Beans and the Entity Beans. Session Beans are objects representing clients in the server side, which can access logic methods such as: image archiving, image groups configuration, image search by attributes, among others. Entity Beans constitute a database abstraction to execute SQL queries and to access relational databases with Java classes. This tier also has the Metadata Manager module to record images and extract textual information such as name, size and DICOM attributes; and the CBIR module, responsible for the feature extraction and similarity operations.

4. Persistent Tier. It provides tools to access the file system where images and their thumbnails are stored as well as the database that contains the metadata information and image features.

5. Security Tier: It provides access control to the application based on the role provided by the JBossSX Security extension, configured in a standard XML descriptor. The security scheme verifies the user rol and allows or refuses the access to some methods or domains.

\footnotetext{
${ }^{3}$ Remote Method Invocation.

4 Java Naming and Directory Interface.
} 

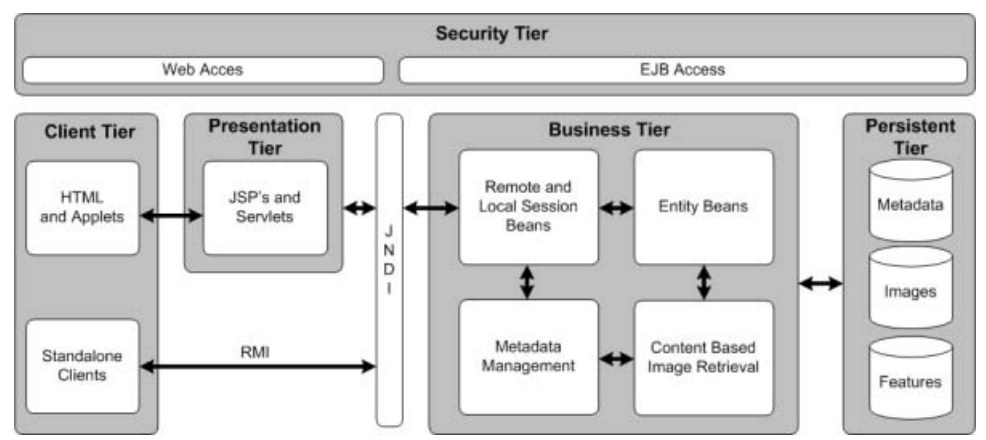

Fig. 2. System global architecture

\subsection{Content-Based Retrieval Module}

One of the main concerns of the proposed architecture is the CBIR module, which is located in the Business tier. The design of this module is based on design patterns which support extension by adding new feature extraction methods and new similarity functions. The CBIR module uses a standarized representation of digital images to apply algorithms independent of the format details. Image features are coded and stored in the Persistent layer to build a content-based index.

The CBIR module has four main submodules: features representation, feature extraction algorithms, similarity functions and retrieval algorithms. The feature representation submodule provides a class hierarchy with a common interface to access features data, and some specializations to handle specific features such as histograms, vectors and trees. The feature extraction submodule uses the Template pattern to codify each feature extraction algorithm in a class method, and associate it to a class in the feature hierarchy. The similarity functions submodule uses a hierarchy of metrics with the Command pattern, allowing an abstract configuration of the parameters to be compared to return the similarity score. The retrieval algorithms submodule, provides a framework to configure different retrieval algorithms with multiple feature-metric pairs and their associated importance factor, with the design of the Observer and Iterator patterns. Every submodule also includes a Factory pattern to dinamically create objects of its hierarchy.

With this structure, it is easy to develop new algorithms for feature extraction and similarity evaluation, making the module reusable and extensible. Currently, implemented features include the histogram features described in section 3, as well as the similarity functions.

\section{Retrieval Performance Evaluation}

Like other information retrieval systems, a CBIR system resolves queries in an approximate way, because users are not aware of the exact results that should be 
delivered [21]. That means that CBIR systems must be evaluated to determine a degree of precision of the retrieval process, revealing how good the system is at locating relevant images associated to the user query. Since the most important contributions of the proposed architecture is the content-based image retrieval module, it is important to assess its performance. In this work a specific evaluation has been made using an experimental dataset of histopathology images, which are used to diagnose a special skin cancer type.

\subsection{Evaluation Framework}

When evaluating information retrieval systems, it is important to define what a perfect system response would be like, named ground truth 22. Many approaches to define a ground truth have been used in the literature, including: user assesment of relevancy; the automatic definition of classifications from available image annotations; and manual definition of user information needs. In this work a ground truth was defined through the analysis, annotation and coding of a set of images, performed manually by pathologists.

The image collection selected to evaluate the system is a database of 6,000 histopathology images, from which a subset of 1,502 images was selected as ground truth. The ground truth, created by pathologists, is composed by 18 general query topics, corresponding to possible information needs in pathology. In average, each query topic has 75 relevant images, and many images are shared by different query topics, i.e. query topics are not disjoint sets, because differential diagnosis patterns could be part of one or many categories.

For the experimental test, each image in the result set is evaluated against the ground truth to see whether it is relevant or not. Müller et al [23] presents a framework to evaluate CBIR systems in order to report comparable results from different research centers in a standarized way. The most representatives of those performance measures are precision and recall:

$$
\begin{gathered}
\text { precision }=\frac{\text { number of relevant retrieved images }}{\text { number of all retrieved images }} \\
\text { recall }=\frac{\text { number of relevant retrieved images }}{\text { number of all relevant images }}
\end{gathered}
$$

This performance measures are easily understandable, and can be taken when different number of images have been retrieved. Another widely used performance evaluation mechanism in CBIR systems is the precision vs recall graph (PR graph), which provides information about the behavior of the system in many points.

\subsection{Experimental Design}

The experimental framework uses the subset of 1,502 annotated images which allows determining whether results are relevant or not. Each experiment is composed of 30 queries (images) randomly selected from the annotated collection. 
When results are obtained, the evaluation procedure verifies if each result belongs to the same query topic of the example image, marking it as relevant or irrelevant.

The goal of this experimentation is to identify features and metrics that output the best results to the user, based on the information of the ground truth. There are different situations to evaluate. Since there are many metrics available, it is important to identify which is the best choice for each feature space. Then, knowing what metric to use for each feature, the test evaluates which featuremetric pair presents the best performance in the general ranking process. In addition, the test verifies whether the combination of different features performs better than individual feature-metric pairs.

\subsection{Results}

The identification of the best metric in each feature space, is determined by the values of precision and recall obtained in the experimentation. Each feature was tested with each metric selecting the best precision rate per feature. Results are shown in Table 1, where features are associated with the metric that outputs the best performance in that space. Reported values correspond to the average precision of the 30 random queries at the first result, e.g. the Sobel-JSD metric returns a relevant image in the fisrt position in the $61 \%$ of the cases. Since those results are the best per feature, i.e. each feature was tested with all metrics and the presented results are the best feature-metric pair, Table 1 also shows features ordered by their precision rate. This test shows that edges, codified into the Sobel Histogram, performs better than any other histogram features, this suggest that edges is an important feature for differential diagnosis in pathology.

Table 1. Average precision values for the best feature-metric pairs

\begin{tabular}{llc}
\hline Feature & Metric & $\mathrm{P}(1)$ \\
\hline Sobel & Jensen-Shannon Divergence & $61 \%$ \\
RGB Histogram & Relative Bin Deviation & $53 \%$ \\
Local Binary Partition & Relative Bin Deviation & $53 \%$ \\
Gray Histogram & Relative Bin Deviation & $50 \%$ \\
Tamura Texture & Euclidean Distance & $39 \%$ \\
Invariant Feature Histogram & Relative Bin Deviation & $36 \%$ \\
\hline
\end{tabular}

Testing the combination of different features, an average precision rate of $67 \%$ was achieved for the first image retrieved. According to the definition 3, a linear combination of features requires the use of different importance factors. In this test, those factors were identified by exhaustive search, finding a combination of $50 \%$ for Local Binary Partition, 30\% for Sobel Histogram and 20\% for RGB Histogram. In the PR graph this configuration outperforms the individual feature-metric pairs previously tested. This tendence is better shown in Figure 3 . in which the combination approach is compared with the best three individual 


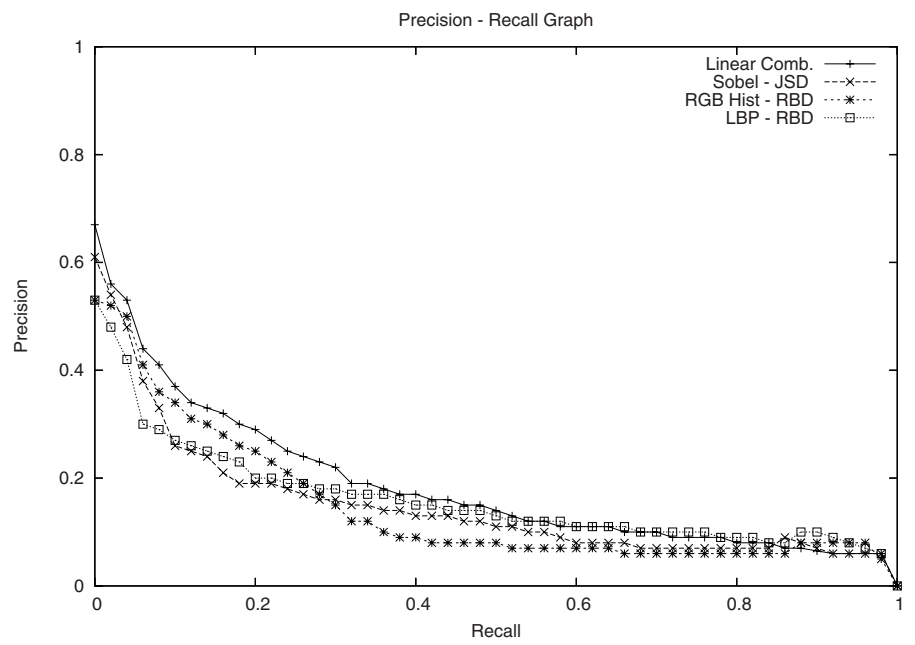

Fig. 3. Precision vs Recall graph comparing the performance of the linear combination approach and other individual metrics

metrics. The linear combination of features shows a better performance than individual features i.e. the precision-recall pairs are the best for the combination of features in almost all the cases.

\section{Conclusions}

This paper has presented the design, development and evaluation of a medical image database system, now in use in the Telemedicine Centre at the National University of Colombias. The proposed system exhibits some particular features that distinguish it from traditional image management systems: its architecture is multi-tier, it provides web access to image collections, and it allows contentbased retrieval.

The content-based-retrieval module provides a search-by-example capability, i.e. the user can retrieve images that are similar to a given reference image. Similar images are retrieved thanks to a two-phase process: feature extraction and similarity evaluation. Different low-level features were implemented including color, texture and edges. Also, different similirity measures were tested, since a given feature, such as a histogram, requieres an appropiate metric.

The content-based-retrieval module was evaluated using a collection of annotated histopathology images. The images were annotated by an especialist stablishing a base line for the system performance. This evaluation demonstrates that some low level features can approximate the differential diagnosis criteria used by pathologists until certain level, which is deemed as adequate for teaching purposes by pathologists who annotated these images. The results may be

5 http://www.telemedicina.unal .edu.co 
outperformed by using high-level features that take into account the semantics of images. The modelling and implementation of these high-level features is part of our future work.

\section{References}

1. Müller, H., Michoux, N., Bandon, D., Geissbuhler, A.: A review of content based image retrieval systems in medical applications clinical bene ts and future directions. International Journal of Medical Informatics 73, 1-23 (2004)

2. Costa, C.M., Silva, A., Oliveira, J.L., Ribeiro, V.G., Ribeiro, J.: A demanding web-based pacs supported by web services technology. SPIE Medical Imaging 6145 (2006)

3. Gutierrez, M., Santos, C., Moreno, R., Kobayashi, L., Furuie, S., Floriano, D., Oliveira, C., João, M., Gismondi, R.: Implementation of a fault-tolerant pacs over a grid architecture. SPIE Medical Imaging - Poster Session 6145 (2006)

4. Chadrashekar, N., Gautham, S.M., Srinivas, K.S., Vijayananda, J.: Design considerations for a reusable medical database. In: IEEE International Symposium on Computer-Based Medical Systems, pp. 69-74 (2006)

5. Marcos, E., Acuña, C., Vela, B., Cavero, J., Hernández, J.: A database for medical image management. Computer Methods and Programs in Biomedicine 86, 255-269 (2007)

6. Caramella, D.: Is pacs research and development still necessary? International Congress Series 1281, 11-14 (2005)

7. Doi, K.: Computer-aided diagnosis in medical imaging: Historical review, current status and future potential. Computerized Medical Imaging and Graphics 31, 198211 (2007)

8. Shyu, C.-R., Brodley, C.E., Kak, A.C., Kosaka, A., Aisen, A.M., Broderick, L.S.: Assert: A physician-in-the-loop content-based retrieval system for hrct image databases. Computer Vision and Image Understanding 75, 111-132 (1999)

9. Lehmann, T.M., Güld, M.O., Thies, C., Plodowski, B., Keysers, D., Ott, B., Schubert, H.: The irma project: A state of the art report on content-based image retrieval in medical applications. Korea-Germany Workshop on Advanced Medical Image, 161-171 (2003)

10. Deselaers, T., Weyand, T., Keysers, D., Macherey, W., Ney, H.: Fire in imageclef 2005: Combining content-based image retrieval with textual information retrieval. Image Cross Language Evaluation Forum (2005)

11. Traina, A.J., Castanon Jr., C.A., C.T.: Multiwavemed: A system for medical image retrieval through wavelets transformations. In: 16th IEEE Symposium on Computer-Based Medical Systems (2003)

12. Tan, Y., Zhang, J., Hua, Y., Zhang, G., Huang, H.: Content-based image retrieval in picture archiving and communication systems. SPIE Medical Imaging - Posters 6145 (2006)

13. Müller, H., Hoang, P.A.D., Depeursinge, A., Hoffmeyer, P., Stern, R., Lovis, C., Geissbuhler, A.: Content-based image retrieval from a database of fracture images. SPIE Medical Imaging 6516 (2007)

14. Lozano, C.C., Kusmanto, D., Chutatape, O.: Web-based design for medical image. In: IEEE International Conference on Control, Automation, Robotics and Vision 3, pp. $1700-1705$ (2002) 
15. Petrakis, E.G.M., Faloutsos, C.: Similarity searching in medical image databases. IEEE Transactions on Knowledge and Data Engineering 9, 435-447 (1997)

16. Nikson, M.S., Aguado, A.S.: Feature Extraction and Image Processing. Elsevier, Amsterdam (2002)

17. Liu, Y., Zhang, D., Lu, G., Ma, W.-Y.: A survey of content-based image retrieval with high-level semantics. Pattern Recognition 40, 262-282 (2007)

18. Deselaers, T.: Features for Image Retrieval. PhD thesis, RWTH Aachen University. Aachen, Germany (2003)

19. Siggelkow, S.: Feature Histograms for Content-Based Image Retrieval. PhD thesis, Albert-Ludwigs-Universität Freiburg im Breisgau (2002)

20. Ashmore, D.C.: The J2EE architect's handbook. DVT Press (2004)

21. Yates, R.B., del Solar, J.R., Verschae, R., Castillo, C., Hurtado, C.: Contentbased image retrieval and characterization on specific web collections. In: Enser, P.G.B., Kompatsiaris, Y., O'Connor, N.E., Smeaton, A.F., Smeulders, A.W.M. (eds.) CIVR 2004. LNCS, vol. 3115, pp. 189-198. Springer, Heidelberg (2004)

22. Müller, H., Rosset, A., Vallee, J.P., Geissbuhler, A.: Comparing features sets for content-based image retrieval in a medical-case database. Medical Imaging 5371, 99-109 (2004)

23. Müller, H., Müller, W., Marchand-Maillet, S., Squire, D.M., Pun, T.: A Framework for Benchmarking in Visual Information Retrieval. International Journal on Multimedia Tools and Applications 22, 55-73 (2003) (Special Issue on Multimedia Information Retrieval) 